\title{
The Medical Care of Multiple Disciplinary Team Reduces the Risks of Complications in Patients after Arteriovenous Fistula Operation
}

\author{
Hong Yang, Zhihua Deng*, Yingxue Zhong, Lianghong Yin, Fanna Liu, Yu Chen\# \\ Department of Nephrology of the First Affiliated Hospital of Jinan University, Guangzhou, China \\ Email: "1139795771@qq.com
}

How to cite this paper: Yang, H., Deng, Z.H., Zhong, Y.X., Yin, L.H., Liu, F.N. and Chen, Y. (2020) The Medical Care of Multiple Disciplinary Team Reduces the Risks of Complications in Patients after Arteriovenous Fistula Operation. Open Journal of Nephrology, 10, 117-124.

https://doi.org/10.4236/ojneph.2020.102012

Received: March 31, 2020

Accepted: May 15, 2020

Published: May 18, 2020

Copyright () 2020 by author(s) and Scientific Research Publishing Inc. This work is licensed under the Creative Commons Attribution International License (CC BY 4.0).

http://creativecommons.org/licenses/by/4.0/

\begin{abstract}
Introduction: Arteriovenous fistula (AVF) operation is recognized a gold standard of hemodialysis for end-stage renal disease. Because various complications may occur during perioperative period, medical care plays an important role in improving the possibility of AVF operation. The objective of the study was to explore the effect of the mode of multiple disciplinary team (MDT) medical care on the patients with end-stage renal disease treated by arteriovenous fistula (AVF) operation. Method: 98 patients with end-stage renal disease under the AVF operation were divided into observation group and control group. The control group was performed with regular medical care and for the observation group was treated under the mode of MDT medical care. Complications after operation, hospitalized time and medical satisfaction were comprehensively compared between the both groups. Results: The overall complications including swelling, bleeding, infection and thrombosis in observation group accounted for 5\%, however, $19 \%$ of patients suffered different complications in the control group. The total complications significantly decreased in the observation group compared with control group $(\mathrm{P}<0.001)$. The hospitalization time was shortened to $9.8 \pm 5.6$ days in the observation group, and the average hospitalization in control group was $17.8 \pm 8.9$ days. The medical satisfaction was increased by 20.83 percent in the observation group compared with the control group $(\mathrm{P}<0.001) .75 \%$ patients in control group were satisfied with medical care, and $98.53 \%$ patients were satisfied for observation group. Conclusion: The mode of MDT medical care was beneficial for patients with end-stage renal disease after AVF operation, which could significantly reduce complications, hospitalized time and improve patients' degree of satisfaction. Thus, it should be recommended in the clinical work.
\end{abstract}




\section{Keywords}

Multiple Disciplinary Team, Medical Care, Hemodialysis, Arteriovenous Fistula

\section{Introduction}

Nowadays, hemodialysis is the main alternative treatment method for patients with end-stage renal disease [1] [2]. Thus, establishing a good extracorporeal circulation pathway is a prerequisite to make it work properly. Currently, AVF operation is usually performed by using autogenous blood vessels and artificial blood vessels. Especially, autogenous blood vessels are preferred in general clinical work, as it is more advantageous than grafts, which can greatly reduce risks of thrombosis and infection in patients with end-stage renal disease [3] [4]. However, it has always been a great challenge for medical staff to make AVF mature and maintain it to exert the optimal effect so as to prolong patient's life. Therefore, How to reduce the postoperative complications, the length of hospitalization, and minimize the medical cost of patients is a great concern for medical staff [5]. Notably, perioperative medical care plays an important role in improving medical quality. The optimal patient management should involve close coordination between specialists and nurses. The MDT mode of treatment is very popular in many medical centers all over the world, however, the mode of medical care is seldom noted in clinical work. Thus, a novel mode of multiple disciplinary team (MDT) medical care was introduced to treat the patients with end-stage renal disease during perioperative period in our department, which transformed the traditional individual empirical medicine into a modern teamwork. This new mode of MDT medical care was based on the multi-discipline and cross-team cooperation, which may reasonably integrate medical resources and provide the best strategy of medical care. In this study, we aimed to determine whether the novel mode of MDT medical care could reduce the postoperative complications, reduce the length of hospitalization, and improve the satisfactions of patients during hospitalization compared with the control group.

\section{Methods}

Ninety-six patients (55 males and 41 females) aged between 18 years and 87 years (mean age, 61.2 years \pm 13.7 years) with end-stage renal disease undergone AVF operation were included in the Department of Nephrology of the First Affiliated Hospital of Jinan University from January 2018 to July 2019 (Table 1). All the patients performed by AVF were equally divided into the observation group and control group for the MDT medical care and regular nursing care respectively according to random sampling principle.

\subsection{MDT Medical Care Procedures}

The members of MDT consist of nephrologists, specialized nurses, vascular 
Table 1. Clinical characteristics of patients with end-stage renal disease $(n=96)$.

\begin{tabular}{cc}
\hline Primary disease & No. Patients, $\mathrm{n}(\%)$ \\
\hline Hypertensive nephropathy & $49(51.04)$ \\
Diabetic nephropathy & $29(30.20)$ \\
Chronic glomerulonephritis & $5(5.20)$ \\
Obstructive kidney disease & $8(8.33)$ \\
Renal cysts & $2(2.08)$ \\
Nephrotic syndrome & $2(2.08)$ \\
IGA nephropathy & $1(1.04)$ \\
\hline
\end{tabular}

surgeons, ultrasound doctors and interventional radiologists. Preoperative examination was completed after the patient's admission. Meanwhile, the transplant surgeon of vascular access group checked the distribution of the patient's arm blood vessels with ultrasound doctors and interventional radiologists preoperatively. Medical care plans were discussed and specified according to the condition of patient under MDT mode. In addition, the MDT members explained the surgical procedures and precautions to patients in detail. Finally, the vascular surgeon made an optimal surgical plan for the patient. The MDT medical care mode for the patients during the perioperative period in detail was as following:

1) Preoperative notification and psychological intervention

A specialized nursing staff pre-educated the patient about the basic knowledge about AVF, the treatment methods, and the risks of common complications after AVF operation so as to make the patient psychologically cooperate with surgical treatment, eliminate tension and fear, which could make the whole treatment progress more smoothly.

2) Preoperative preparation

The patient was instructed to wash the surgical area with soap 3 times, and bathe the day before operation, moreover, the patient's vital signs and blood glucose changes were recorded and reported to the vascular surgeon if there is any abnormality.

3) Post-operative medical care

On the first day after AVF operation, the specialized nursing staff recorded the patient's vital signs, inquired the patient about the symptoms of discomfort, and taught the patient to raise the arm appropriately to promote blood return and reduce swelling and bleeding of the limb on the side of operation. Within three days after the operation, the patient was instructed not to flex excessively in case of thrombosis and hematoma occurrence in the operation area. Meanwhile, it was forbidden for medical staff to measure blood pressure, draw blood, intravenous injection, and blood transfusion on the side limbs of the internal fistula. Moreover, the patient was told to wear loose cuffs as much as possible and monitor the internal fistula noise at least 3 times a day.

4) Daily internal fistula care

The patient performed AVF operation should keep the skin clean, trim the 
nails regularly in case of accidental injuries of internal fistula limbs of fistula after discharge. If the wound is free of bleeding, infection, the internal fistula limb could be used daily. In addition, the patient should measure blood pressure in time, and soak the limbs of the internal fistula with warm water appropriately on a certain time. Follow-up was necessary to prevent blockage of AVF. Finally, it was forbidden for patients to lift weights over $5 \mathrm{~kg}$ and prevented compression of AVF.

\subsection{Regular Medical Care Procedures}

On the contrary, the patients in control group were administered with routine medical care in our nephrology department. A nurse in charge was responsible for the patients' admission education, physical examination, postoperative care, functional exercise, and discharge education for patients. Then, the vascular surgeon performed AVF operation after assessing the patients' examination results from ultrasound and imaging departments. A follow-up inquiry was made for each patient after discharge on a certain time by telephone.

\subsection{Clinical Quality Survey}

1) Postoperative complications Postoperative complications include swelling, bleeding, infection, and thrombosis was precisely recorded for each patient.

2) Length of hospitalization The average hospitalized time of the patients in two groups was compared.

3) Medical satisfaction survey The medical satisfaction survey was conducted by telephone questionnaire as a part of follow-up. Satisfaction degree was classified according to the feedback of the patients as following: very satisfied: 80 to 100 scores; satisfaction: 60 to 79 scores; dissatisfaction: $\leq 59$ scores.

Medical satisfaction survey of patient in the nephrology department of Jinan University

\begin{tabular}{l} 
Telephone Questionnaire \\
\hline 1) did doctors and nurses politely take medical history? \\
2) did doctors and nurses tell you the course of operation in detail? \\
3) were you satisfied with physical examinations and medical care? \\
4) did doctors and nurses tell you the complications that might \\
occur and how to prevent them postoperatively? \\
5) did doctors and nurses instruct you precautions and provide \\
psychological intervention preoperatively? \\
6) did doctors and nurses protect your privacy strictly? \\
7) did doctors and nurses teach you how to protect the fistula \\
and get recovered? \\
8) did doctors or nurses perform follow-up in time? \\
9) were you satisfied with your therapy? \\
10) did doctors or nurses deal with the feedback from you in time?
\end{tabular}




\subsection{Statistical Analysis}

The Clinical quality survey data were statistically analyzed by SPSS_21.0 software. The two-independent-samples tests or $\chi 2$ test was used for analysis between the two groups. $\mathrm{P}<0.05$ was considered statistically significant.

\section{Results}

Out of 96 patients with end-stage renal disease, 49 had Hypertensive nephropathy as to say a prevalence of $51.04 \%$. Twenty-nine patients had diabetic nephropathy and 5 had chronic glomerulonephritis. Eight patients had past medical history of Obstructive kidney disease, 2 patients had renal cysts and 2 patients suffered from nephrotic syndrome. Only 1 patient was diagnosed as IGA nephropathy.

\subsection{Postoperative Complications}

The two-independent-samples tests showed that the complications in the observation group were significantly lower than those in the control group, and the difference was statistically significant $(P<0.001)$. In our study, swelling was found in 6 patients $(12.5 \%)$ in the control group and only 2 patients had swelling in the observation group (4.2\%). Bleeding was noted in 8 patients and 3 patients in the control group (16.7\%) and observation group (6.3\%) respectively. There were 2 patients $(4.2 \%)$ and 3 patients (6.3\%) underwent infection and thrombosis respectively in the control group. On the contrary, infection and thrombosis were not found in the observation group (Table 2).

\subsection{Duration of Hospitalization}

The length of hospitalization in the two groups was recorded and analyzed using t-test. It showed that the length of stay in the observation group was significantly shorter than that in the control group $(\mathrm{P}<0.001)$. The hospitalization time was shortened to $9.8 \pm 5.6$ days in the observation group, and the average hospitalization in control group was $17.8 \pm 8.9$ days (Table 3 ).

\subsection{Medical Satisfaction Survey}

$75 \%$ patients in control group were satisfied with medical care, and $98.53 \%$ patients were satisfied for observation group. The satisfaction degree of patients in

Table 2. The comparison of complications between two groups (n, \%).

\begin{tabular}{ccc}
\hline Complications & Control group & Observation group \\
\hline swelling & $6(12.5)$ & $2(4.2)$ \\
bleeding & $8(16.7)$ & $3(6.3)$ \\
infection & $2(4.2)$ & $0(0)$ \\
thrombosis & $3(6.3)$ & $0(0)$ \\
total & $19(38)$ & $5(12)$ \\
Mann-Whitney U & 816 & $\mathrm{P}<0.001$ \\
\hline
\end{tabular}


Table 3. The comparison of length of hospitalization between the two groups.

\begin{tabular}{cc}
\hline group & Average stay (day) \\
\hline Control group & $17.8 \pm 8.9$ \\
Observation group & $9.8 \pm 5.6$ \\
$\mathrm{t}$ & 5.453 \\
$\mathrm{P}$ & $<0.001$ \\
\hline
\end{tabular}

the observation group was significantly higher than that in the control group ( $\mathrm{P}$ $<0.001$ ) (Table 4).

\section{Discussion}

AVF is considered the gold standard modality of vascular access for maintaining hemodialysis because of its superior patency, provision of high quality of life, and low risk of patient mortality [6]. However, some of complications after AVF operation, including immediate thrombosis, failing to mature, swelling or infection, might occur [7] [8]. As the patency and function of the AVF is related to the prognosis and quality of life of the patient [9], a good operation plan is very important for the success of AVF surgery, however, the considerable medical care is also indispensable for improving the medical quality, especially in the aspect of reducing postoperative complications. However, the traditional medical care mode was gradually unable to meet the growing medical needs of patients, and the MDT medical care mode is a new type of exploration used by clinical institutions at present, and has achieved unexpected good results.

Compared with the conventional medical care, the application of MDT mode could significantly reduce the complications postoperatively. The Duplex ultrasound identifies suitable arteries and veins for successful AVF creation [10]. Prognosis regarding the efficiency of a fistula is made with the help of duplex ultrasound, an instrument that enables evaluation of the success of fistula development [5] [11]. In addition, early detection and intervention can save the fistula when complications occur, especially for the diagnosis of thrombosis or swelling [12]. It is found that inflammation caused by postoperative infection is also involved in AVF thrombosis [13]. Therefore, how to reduce the complications after the AVF operation is very critical to the survival of the patients with end-stage renal disease. We could receive all kinds of feedback and suggestions from patients and instruct them to avoid different complications. Meanwhile, the MDT mode increased the sense of responsibility of medical staff, and improved the team spirit of cooperation between different departments and played advantage to maximum. Patients could cooperate with the guidance of medical staff and actively perform postoperative functional exercises, so that patients could do self-adjustment and find abnormal conditions in time.

In the control group, most patients complained lack of systemic instruct about how to restore the function of AVF. However, there were only several patients complained the complicated clinical examination. Most patients under MDT 
Table 4. The medical satisfaction survey between two groups of patients (n, \%).

\begin{tabular}{ccccc}
\hline group & very satisfied & satisfied & less satisfied & overall satisfaction (\%) \\
\hline Control group 12 & 26 & $1036(75)$ & & \\
Observation group & 43 & 3 & 2 & $46(95.83)$ \\
& & $\chi^{2} 43.464$ & & \\
& $\mathrm{P}<0.001$ & \\
\hline
\end{tabular}

medical care were greatly satisfied with the good service and quick feedback from doctors and nurses. Under the novel MDT medical care mode, patient satisfaction degree has improved significantly, which greatly reduced the occurrence of doctor-patient contradictions, and made medical care work more smoothly. Consequently, the whole medical work entered a virtuous circulation mode and realized seamless connection between different departments. To be noted, the decreases of patients' complications significantly shortened the length of patient hospitalization and reduced medical costs. Therefore, the MDT mode of medical care can better provide patients with high-quality services, thereby achieve good effects on treatment and medical care.

\section{Limitation of the Study}

MDT mode of medical care is a new trial in clinical work, which needs more team-spirit and cooperation. This may be an obstacle to the application of MDT mode. Second, the MDT mode of medical care should be optimized to reduce medical costs.

\section{Conclusion}

The MDT medical care mode focuses on the specialties of nephrologists, vascular access group doctors, ultrasound doctors, and interventional surgeons, and provides a simple and easy-to-use medical system based on disciplines such as laboratory medicine and imaging. Optimizing the allocation of resources greatly shortens the average hospital stay of patients, reduces the medical expenses and financial burden on patients, and improves high medical satisfaction. Therefore, the multi-disciplinary medical nursing mode is scientific and effective in current nursing work, and it is worthy of popularization and application in clinical work.

\section{Acknowledgements}

We thank all the staff of the Department of Nephrology of the First Affiliated Hospital of Jinan University. This research was supported by grant 2013B090400004 from Guangzhou Academic foundation of the Industry-Academy-Cooperation, China.

\section{Conflicts of Interest}

All authors have no conflicts of interest to declare. 


\section{References}

[1] (2006) Clinical Practice Guidelines for Vascular Access. American Journal of Kidney Diseases, 48, S176-S273. https://doi.org/10.1053/j.ajkd.2006.04.040

[2] Konner, K., Nonnast-Daniel, B. and Ritz, E. (2003) The Arteriovenous Fistula. Journal of the American Society of Nephrology, 14, 1669-1680. https://doi.org/10.1097/01.ASN.0000069219.88168.39

[3] Lee, T., Barker, J. and Allon, M. (2007) Comparison of Survival of Upper Arm Arteriovenous Fistulas and Grafts after Failed Forearm Fistula. Journal of the American Society of Nephrology, 18, 1936-1941. https://doi.org/10.1681/ASN.2006101119

[4] Allon, M., Lockhart, M.E., Lilly, R.Z., Gallichio, M.H., Young, C.J., Barker, J., Deierhoi, M.H. and Robbin, M.L. (2001) Effect of Preoperative Sonographic Mapping on Vascular Access Outcomes in Hemodialysis Patients. Kidney International, 60, 2013-2020. https://doi.org/10.1046/j.1523-1755.2001.00031.x

[5] Manns, B., Tonelli, M., Yilmaz, S., Lee, H., Laupland, K., Klarenbach, S., Radkevich, V. and Murphy, B. (2005) Establishment and Maintenance of Vascular Access in Incident Hemodialysis Patients: A Prospective Cost Analysis. Journal of the American Society of Nephrology, 16, 201-209. https://doi.org/10.1681/ASN.2004050355

[6] Nanami, M., Suemitsu, K., Nagasawa, Y., Hasuike, Y., Kuragano, T. and Nakanishi, T. (2019) Current Topics in Vascular Access: Superficialization of Arteriovenous Fistula. Contributions to Nephrology, 198, 1-11. https://doi.org/10.1159/000496530

[7] Mercado, C., Salman, L., Krishnamurthy, G., et al. (2008) Early and Late Fistula Failure. Clinical Nephrology, 69, 77-83. https://doi.org/10.5414/CNP69077

[8] Bozzetto, M., Brambilla, P., Rota, S., et al. (2018) Toward Longitudinal Studies of Hemodynamically Induced Vessel Wall Remodeling. The International Journal of Artificial Organs, 41, 714-722. https://doi.org/10.1177/0391398818784207

[9] Lorenzo, V., Martin, M., Rufino, M., Hernández, D., Torres, A. and Ayus, J.C. (2004) Predialysisnephrologic Care and a Functioning Arteriovenous Fistula at Entry Are Associated with Better Survival in Incident Hemodialysis Patients: An Observational Cohort Study. American Journal of Kidney Diseases, 43, 999-1007. https://doi.org/10.1053/j.ajkd.2004.02.012

[10] Siddiqui, M.A., Ashraff, S. and Carline, T. (2017) Maturation of Arteriovenous Fistula: Analysis of Key Factors. Kidney Research and Clinical Practice, 36, 318-328. https://doi.org/10.23876/j.krcp.2017.36.4.318

[11] Mendes, R.R., Farber, M.A., Marston, W.A., Dinwiddie, L.C., Keagy, B.A. and Burnham, S.J. (2002) Prediction of Wrist Arteriovenous Fistula Maturation with Preoperative Vein Mapping with Ultrasonography. Journal of Vascular Surgery, 36, 460-463. https://doi.org/10.1067/mva.2002.126544

[12] Lew, S.Q., Nguyen, B.N. and Ing, T.S. (2015) Hemodialysis Vascular Access Construction in the Upper Extremity: A Review. The Journal of Vascular Access, 16, 87-92. https://doi.org/10.5301/jva.5000299

[13] Kaysen, G.A. and Don, B.R. (2003) Factors That Affect Albumin Concentration in Dialysis Patients and Their Relationship to Vascular Disease. Kidney International, 84, S94-S97. https://doi.org/10.1046/j.1523-1755.63.s84.20.x 